\title{
Research on SOA based GIS service for smart distribution system
}

\author{
Xi Zhang ${ }^{1}$, Taifeng Kang ${ }^{2, a^{*}}$, Xiaoping Xie $^{2}$, Fengqing Zhao ${ }^{2}$, Shan Tang ${ }^{1}$ and \\ Hu Huang ${ }^{1}$ \\ ${ }^{1}$ State Grid Chongqing Electric Power Company Nan'an Power Supply Company, Chongqing, \\ China \\ ${ }^{2}$ Beijing Sifang Automation CO., LTD., Beijing, China \\ aarfa2011_1@163.com \\ ${ }^{*}$ Corresponding author
}

Keywords: service oriented architecture (SOA); geographic information system (GIS); tile map; outline sketch; distribution management system (DMS); smart distribution system

Abstract. Distribution system is the section to deliver power energy to end user and realize power consumption, which draws more and more attentions. In the daily life of system operation and control for smart distribution system, the system operators heavily rely on Geographic Information System (GIS). Traditional GIS based applications and systems are limited in isolated systems. This paper presents a SOA based framework for applications in smart distribution system to integrate with GIS services. In the framework, SOA based GIS service and applications are created to integrate other system such as DMS with GIS. The method proposed in this paper can integrate the GIS service into DMS to create advanced applications and provide essential human-computer interaction for the operation and management of smart distribution system. The proposed method of this paper has been applied to actual projects and achieves good results.

\section{Introduction}

In modern society, efficient, secured and reliable power supply has become more and more important. Distribution system is the section to deliver power energy to end user and realize power consumption, which draws more and more attentions. In order to increase the efficiency and reliability of power delivery in distribution system, smart distribution system has been promoted as future distribution system [1]. In the daily life of operation and control for smart distribution system, the system operators heavily rely on GIS[2], which provides convenient computer-aided and decision-making applications [3, 4].

Traditional GIS based professional applications and systems are directly built on commercial GIS platform. In this case, these applications are tightly coupled with the GIS platform, which could provide good performance and efficiency. However, these applications are limited in the isolated system. Applications reusing and data sharing are nearly impossible and in low efficiency. As a result, many applications and systems are developed redundantly. The tools for operation and management of distribution system and smart distribution system are usually based on display with geographic information. As GIS and DMS are separate and isolated systems, display with the geographic information cannot be directly utilized by DMS. Some operation with geographic information such as equipment remote control and abnormal tag tipping cannot be accomplished in either GIS or DMS directly.

Loose coupled applications and systems with GIS platform are the trends for the development of GIS applications and systems in specialty and professional categories [5]. This paper presents an SOA based framework for applications in smart distribution system to integrate with GIS services. In the framework, SOA based service interfaces and applications are created to provide GIS based services to DMS applications. The method proposed in this paper can integrate DMS with GIS service with and develop advanced applications, providing the fundamental and efficient human-computer interactions for the operation of smart distribution system. The research results of this paper have been applied to the operation and management of actual distribution network, and achieve good results. 


\section{Requirements of GIS for distribution system operation and management}

Smart distribution system. Smart distribution network is to realize wide area system supervision and remote control as well as system operation optimization to provide secured, efficient and economical power energy to end users by utilizing intelligent measurement and communication technologies and integrating with massive system resources such as distributed generators, energy storage devices and electric vehicles.

The requirements for GIS in operation and management of smart distribution system. To provide secured, efficient and economical power energy to end users, system operators need to fast locate and display equipments on geographical map, investigate on abnormal operation state, realize power service restoration, etc. These operations and managements are mostly accomplished by integration of GIS and DMS. In general, the requirements can be summarized as locating and positioning of specific devices, synchronizing display with diagrams and geographic information as well as operation and decision support with geographic information.

Locating and positioning of specific devices. When system operators check or change operation status of specific equipment, they usually use diagrams as they show the connectivity more suitable for logical thinking. However, there is simultaneous requirement for locating the equipment on geographic map. Besides equipment investigation, the abnormal status of the equipment is also important. The various kinds of abnormal status, such as outage, fault, on the loop, maintenance, overload, which could be tagged on the diagrams in DMS, will be very useful to be displayed on the corresponding equipment on geographic map.

Synchronizing display with diagrams and geographic information. As there are two types of display, the single line diagram and the geographic map, the system operators could investigate on or change the system operation status from either display. These two types of display should be synchronized and consistent. The operators could operate on one display, and the other display should locate on the same district or equipments simultaneously with the latest status.

Operation and decision support with geographic information. The equipments on the geographic map should have situation perception ability so as to perform specific control or simulate operation based on the geographic map. And the results of such control or simulate should be updated on the geographic map.

\section{SOA based method to integrate applications with GIS system for smart distribution system}

Service oriented architecture. SOA is a methodology for constructing distributed software systems to provide a uniform ways for resource discovery, information sharing and capabilities integration by multiple complex enterprise systems.

GIS service for smart distribution system. SOA based GIS service integrates different modules in GIS systems to provide various functions in standard services to other systems with geographic information. According to previous analysis on the requirement of DMS and smart distribution system for GIS, these services can be summarized as tile mapping service, outline sketch service, positioning service, and layer management service and synchronization service. The architecture of GIS service for smart distribution system is shown in Fig. 1.

Tile mapping service. Tile mapping service is the basic service to provide the geographic background for DMS to realize geographic presentation of equipments. In this service, it launches the request for the tile map service to the GIS in different scaled levels. As GIS returns the geographic information required, the tile maps are cached into the directory of DMS, and they are showed as background when there is the need to present on geographic map. By caching the specific geographic background, unnecessary delay caused by the large amount of data transmission is avoided, which improves the efficiency of geographic information access.

Outline sketch service. The outline sketch service shows the trend and the outline of the equipment (usually a feeder or a group of feeders) on the geographical background which is essential to display equipment information. The outline sketch is request for GIS system based on feeders, and the 
geographic information is converted as graphs into DMS. DMS also calculates the coordinates of the outline sketch of the feeder and saves the coordinates. The graphs and coordinates are used together to display the outline sketch of feeders.

Positioning service. When positioning of a equipment in DMS, the related feeder is found out, then the outline sketch of the feeder is launched and the equipment is positioned in the middle of the display. According to the geographic coordinates of the graphic area, a new layer is set up to load the corresponding geographical background tile map. This service launches both the tile map and the outline sketch.

Layer management service. Besides tile map as background and the outline sketch for the top display, there is need to provide graphical information such as measurements mapping or abnormal status tagging. Such information is provided based on multiple layers. Layer management service is used to manage these layers and synchronize between displays when operation occurs either on the single line diagram or on the geographic map.

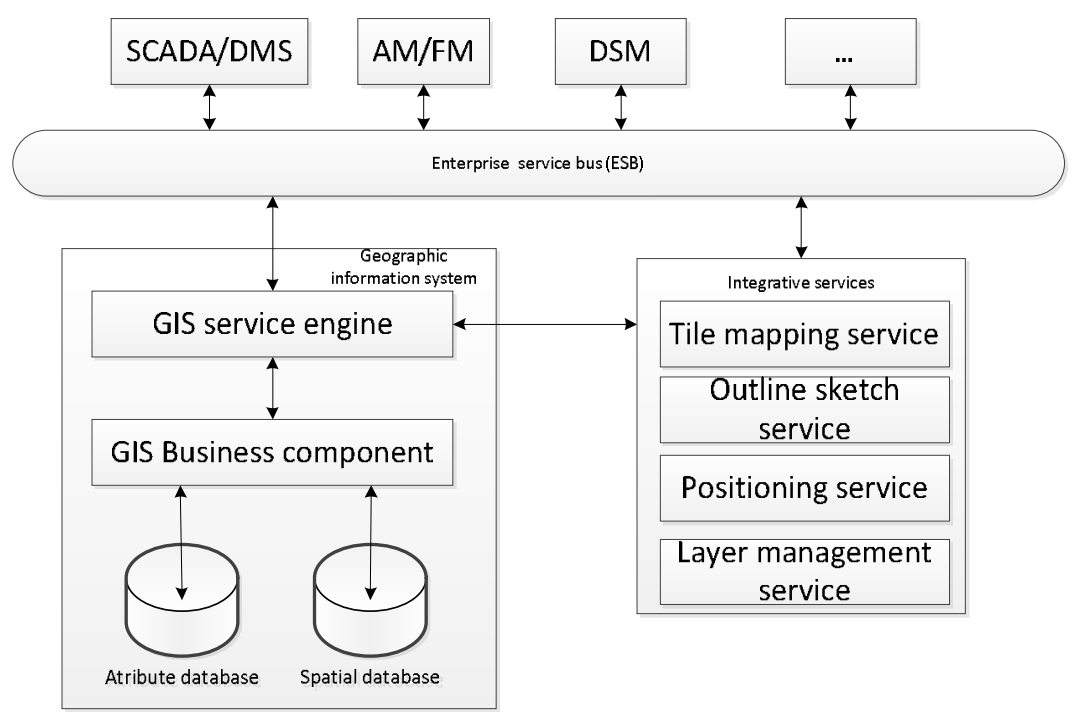

Fig. 1 The framework of SOA based services for integrating GIS to DMS

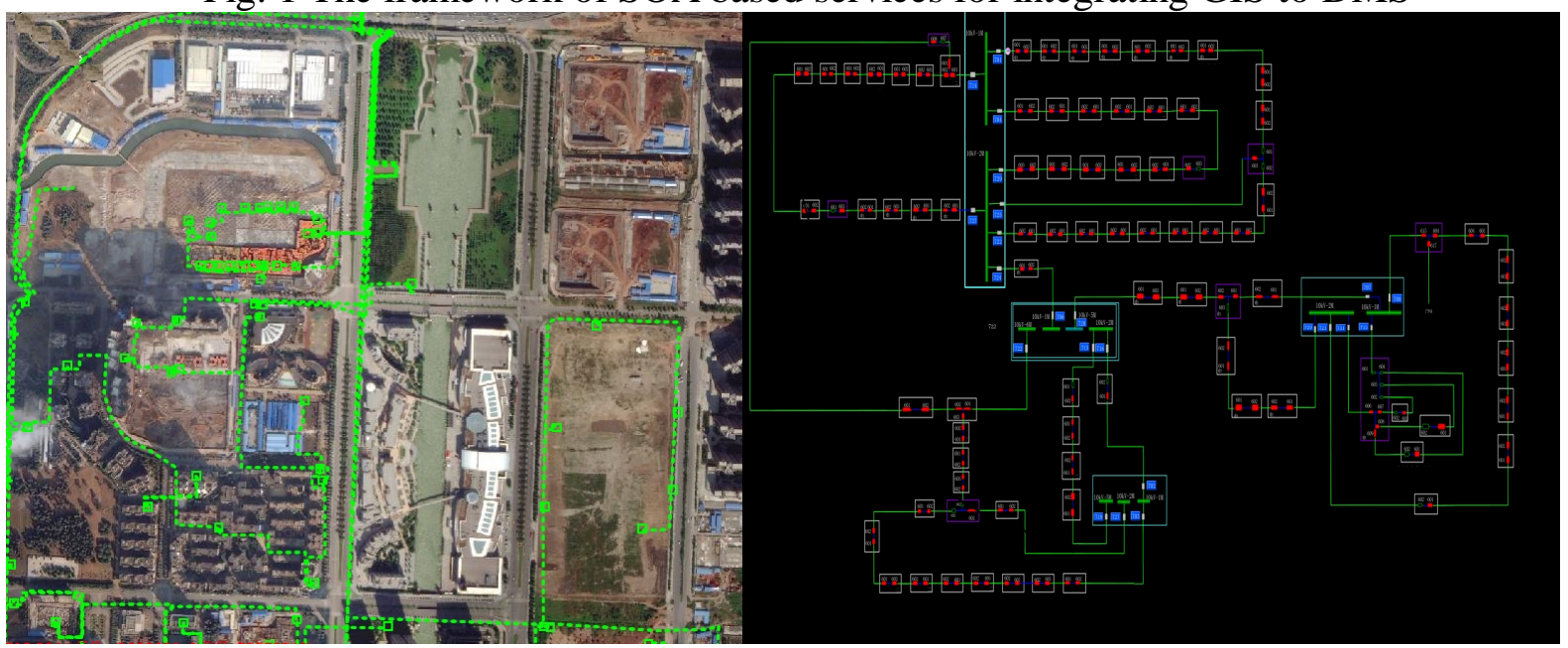

Fig. 2 The example of SOA based services for integrating GIS to DMS

\section{Case study}

We have built up DMS applications integrated with GIS services based on our proposed method in actual projects. Fig. 2 is an example in actual projects for presenting distribution system on geographic background with our proposed method. In this example, a region of distribution system is presented both as the single line diagram (right part of the figure) and as outline sketch with geographic background (left part of the figure). We can simultaneously locate the feeders and circuit breakers on 
geographic background by selecting the equipments on the single diagrams. The views of single line diagram and geographic display are synchronized. Advanced functions are developed on both views, such as abnormal status tags tipping, remote control, system operation and simulation, etc.

\section{Summary}

Geographic information is important for operation and management of smart distribution system as it provides direct and intuitional vision of the equipments in the distribution system. In this paper, we research on the SOA based framework to integrate geographic information from GIS into DMS applications. With the proposed framework, we realize to present equipments with outline sketch on geographic background as well as abnormal status tags tipping to provide decision support. The method is fast, applicable and easy accomplished to integrate DMS applications with GIS functions. Example of actual projects has shown its advantages.

\section{References}

[1] Bingyin Xu, Tianyou Li and Yongduan Xue: Automation of Electric Power Systems, 33(17) (2009):38-41.

[2] Goodchild M F: International Journal of Geographical Information Science, Vol.6 (1992) 31-45.

[3] Weng Yingjun, Zhu Zhongying: Automation of Electric Power Systems, 27(18) (2003):74-78

[4] Liu Yaxin, Xia Li: Power System Technology, 31(16) (2007):42-45.

[5] Yuan Soe-Tsyr, Lu Mei-Rung: Expert Systems with Applications, 36(2009):3671-3694. 